\title{
Prevalence of Hepatitis C Virus in an Endemic Area of Thailand: Burden Assessment toward HCV Elimination
}

\author{
Rujipat Wasitthankasem, ${ }^{1,2,3}$ Napaporn Pimsingh, ${ }^{4}$ Khuandao Treesun, ${ }^{4}$ Nawarat Posuwan, ${ }^{1}$ Preeyaporn Vichaiwattana, ${ }^{1}$ \\ Chompoonut Auphimai, ${ }^{1}$ Ilada Thongpan, ${ }^{1}$ Sissades Tongsima, ${ }^{2,3}$ Sompong Vongpunsawad, ${ }^{1}$ and Yong Poovorawan ${ }^{1 *}$ \\ ${ }^{1}$ Center of Excellence in Clinical Virology, Faculty of Medicine, Chulalongkorn University, Bangkok, Thailand; ${ }^{2}$ National Biobank of Thailand, \\ National Science and Technology Development Agency, Pathum Thani, Thailand; ${ }^{3}$ National Center for Genetic Engineering and Biotechnology, \\ National Science and Technology Development Agency, Pathum Thani, Thailand; ${ }^{4}$ Non-Communicable Disease Control Group, Phetchabun
} Provincial Health Office, Phetchabun, Thailand

\begin{abstract}
Chronic hepatitis $\mathrm{C}$ virus (HCV) infection can lead to liver cirrhosis and hepatocellular carcinoma. To eliminate HCV infection in an endemic area, an epidemiological baseline of the current HCV infection in the population is required. We therefore aimed to evaluate the HCV burden in the Thai Province of Phetchabun, which has the highest HCV infection rate in the country. Toward this, a province-wide district-based representative sampling of 4,769 individuals ages 35-64 years previously shown to represent high-risk age-groups were tested for anti-HCV antibodies using the automated chemiluminescent microparticle assays. Active HCV infection and subsequent genotyping were determined from serologically reactive samples by amplification of the HCV core gene. We found that $6.9 \%(327 / 4,769)$ were anti-HCV positive, of which $75.8 \%$ (248/327) had detectable HCV RNA and 5.8\% (19/327) were in the presence of hepatitis B virus coinfection. Nucleotide sequencing and phylogenetic analysis revealed that HCV genotype 6 was the most prevalent (41\%, 101/248), followed by genotype 3 (31\%, 78/248), and genotype 1 (28\%, 69/248). Socioeconomic and demographic factors including male gender, education, and agricultural work were associated with HCV seropositivity. From these results, we defined the regional HCV genotypes and estimated the HCV burden necessary toward the implementation of pan-genotypic direct-acting antivirals, which may be appropriate and effective toward the diversity of genotypes identified in this study. Micro-elimination of HCV in Phetchabun may serve as a model for a more comprehensive coverage of HCV treatment in Thailand.
\end{abstract}

\section{INTRODUCTION}

Approximately 71 million people worldwide are chronically infected with the hepatitis $\mathrm{C}$ virus (HCV). ${ }^{1}$ It is a major contributor to morbidity and mortality that is projected to exceed the total deaths from HIV infection, tuberculosis, and malaria combined. ${ }^{2}$ Effective therapy using direct-acting antivirals (DAAs) is orally administered and well tolerated. Treatment duration is short and can achieve $>90 \%$ cure rate. With the possibility of a foreseeable elimination of viral hepatitis, the WHO aims to reduce new cases of chronic HCV infection by $90 \%$, decrease $\mathrm{HCV}$-associated mortality by $65 \%$, and treat $80 \%$ of chronic HCV infection. ${ }^{2,3}$ To attain these objectives, however, HCV diagnostic rate and treatment access would need to be increased.

The National Health Security Office within the Thai Ministry of Public Health adopted a pegylated-interferon (IFN) therapy for HCV infection beginning in 2012. Treatment access for patients, however, remains limited because of the stringent eligibility criteria. As such, only a small number of patients with chronic HCV have been treated. In many countries, IFN regimen is no longer the standard therapy and all HCV-infected patients are treated with DAAs; however, HCV treatment with pan-genotypic DAAs has not been implemented in Thailand. Preliminary evaluation of the existing HCV burden in Thailand shows that approximately $760,000-790,000$ Thais are HCV seropositive, half of whom are living with chronic infection. ${ }^{4-6}$ Nevertheless, the prevalence of viral hepatitis appears to be on the decline nationally as a result of fewer new HCV

\footnotetext{
*Address correspondence to Yong Poovorawan, Center of Excellence in Clinical Virology, Faculty of Medicine, Chulalongkorn University, 1873 Rama Rd., Pathumwan, Bangkok 10330, Thailand.
} E-mail: yong.p@chula.ac.th infections, improved blood donation screening, awareness of blood-borne pathogens, and decreasing number of intravenous drug users (IVDUs). ${ }^{5,7}$ Preventing HCV-related morbidity and mortality should therefore contribute to improved quality of life and significant savings in future healthcare costs.

Previously, we have defined a substantially high HCV seropositivity $(15 \%)$ and rate of viremia $(12 \%)$ among adults aged 35 years or older in some areas of Phetchabun Province in Thailand compared with the rest of the nation. ${ }^{8-10}$ Factors such as male gender, educational status, agriculture work, blood transfusion, IVDUs, and tattoos were found to be associated with anti-HCV positivity. ${ }^{8,10}$ Because of the considerable burden of HCV infection, this province was given a priority in the implementation of a sweeping HCV elimination program. To guide the national policy toward an effective treatment program, we sought to characterize the seroprevalence, active carrier rate, and the circulating HCV genotypes in the whole province. This information will be important toward implementing the HCV micro-elimination program in an endemic area and evaluating the potential feasibility of a pointof-care DAA treatment program.

\section{MATERIALS AND METHODS}

Study population. Phetchabun Province has an overall population of 995,000, and most residents are engaged in farming and other agriculture work. ${ }^{8,11}$ This province is composed of 11 administrative districts collectively spanning approximately $300 \mathrm{~km}$ from north to south. ${ }^{12}$ They are further subdivided into 117 subdistricts, each of which is served by at least one primary healthcare center. Enrollment took place from August through September 2018. For every 100 individuals residing in a subdistrict, one person underwent purposive sampling by the primary healthcare center for inclusion 
in this study (Supplemental Table 1). The inclusion criteria were adult aged 35-64 years (representing approximately 438,000 residents) with no apparent underlying immunodeficiency disorders, chronic diseases, HIV infection, or prior treatment for HCV. Individuals with a history of immunosuppressive therapy or clinical signs of chronic liver disease from hepatitis B virus (HBV) or HCV infection were also excluded. The Institutional Review Board of the Faculty of Medicine of Chulalongkorn University approved this cross-sectional descriptive study (Institutional Review Board no. 266/61). Participants were informed of the study objective and consented to take part in the study. The nursing staff at the primary healthcare centers obtained blood samples and basic demographic information, which were sent to their respective district hospitals for processing. In all, there were 4,769 individuals in this study (1,466 men and 3,303 women).

Serological assay. Plasma samples were tested for antiHCV antibodies at the Center of Excellence in Clinical Virology in Bangkok by using an automated chemiluminescent microparticle immunoassay (ARCHITECT anti-HCV assay, Abbott Diagnostics, Chicago, IL) according to the manufacturer's instructions. Anti-HCV results were based on the signal-to-cutoff ratio (S/CO) in which $\mathrm{S} / \mathrm{CO} \geq 1.00$ was automatically assigned as reactive by the Architect i1000SR platform. Reactive samples were further tested for HBV surface antigen using the Architect HBVsAg assay (Abbott Diagnostics).

Nested reverse transcription-polymerase chain reaction (RT-PCR) and genotyping. For all anti-HCV-reactive samples, viral RNA was extracted from $200 \mu \mathrm{L}$ plasma using an Automatic Nucleic Acid Extraction System GENTi 32 (GeneAll Biotechnology, Seoul, Korea). Complementary DNA was synthesized from $10 \mu \mathrm{L}$ of extracted RNA using a HighCapacity cDNA Reverse Transcription Kit (Applied Biosystems, Foster City, CA) according to the manufacturer's instructions. Nested RT-PCR was performed to amplify the partial HCV core gene by using the AccuStart II GelTrack PCR Super Mix (QuantaBio, Beverly, MA). For the first-round PCR, primers 954F (5'-ACTGCCTGATAGGGTGCTTGCGAG-3', nucleotide position 288-311 based on a reference strain with GenBank accession number M62321) and 410R (5'-ATG TACCCCATGAGGTCG GC-3', position 732-751) were used. For the second-round PCR, primers 953F (5'-AGGTCTCG TAGACCGTGCATCATG-3', position 321-344) and 951R (5'CACTGTRAGGGTATCGATGAC-3', position 705-725) were used. The PCR conditions were pre-incubation at $95^{\circ} \mathrm{C}$ for 3 minutes, 40 cycles of denaturation at $95^{\circ} \mathrm{C}$ for 1 minute, annealing at $49^{\circ} \mathrm{C}$ for 1 minute, extension at $72^{\circ} \mathrm{C}$ for 90 seconds, and final extension at $72^{\circ} \mathrm{C}$ for 7 minutes. Amplicons of approximately 400 base pairs were purified and subjected to Sanger sequencing. Nucleotide sequences were analyzed by using BLASTN (http://www.ncbi.nlm.nih.gov), and final genotypes were determined from phylogenetic analysis. Neighbor-joining trees were generated using the Kimura's two-parameter nucleotide substitution model with 1,000 bootstrap tests. Genotype was assigned according to the respective reference genotype clustering clade (genotype 1a: M62321 and M67463, 1b: D90208 and M58335, 1c: D14853 and AY051292, 2a: D00944, AB047639, and D10988, 2b: AB030907, 2c: D50409, 3a: D17763 and D28917, 3b: D49374, 4a: Y11604, 5a: Y13184, 6a: Y12083 and AY859526, 6b: D84262, 6c: EF424629, 6d: D84263, 6e: DQ314805, 6f:
DQ835760, 6g: D63822, 6h: D84265, 6i: DQ835770, 6j: DQ835769, 6k: D84264, 6l: EF424628, 6m: DQ835767, 6n: DQ278894, 6o: DQ835768 and EF424627, 6p: EF424626, 6q: EF424625, 6r: EU408328, 6s: EU408329, 6t: EF632071 and EU246939, 6u: EU246940, 6v: EU158186 and EU798760, 6w: DQ278892 and EU643834, 6xa: EU408330 and EU408332, and 7a: EF108306). ${ }^{13}$ The nucleotide sequences of HCV from this study were submitted to the GenBank database under the accession numbers MN451395-MN451642.

Data analysis. Differences between groups of categorical variables (gender, geography, and age-group) were analyzed by the chi-square test. The logistic regression model evaluated the association between anti-HCV-positive status and demographic parameters (gender and age). Odds ratios (ORs) with $95 \%$ Cls were derived from univariate and multivariate analyses. A $P$-value $<0.05$ was considered statistically significant. Statistical analysis was performed using SPSS Statistics version 22 (IBM Corporation, Armonk, NY). From the anti-HCV and HCV RNA prevalence found in this study, we estimated the HCV seropositivity and viremic carriers in Phetchabun by extrapolation from the age-stratified population census registry retrieved from the Health Data Center database of Thailand. ${ }^{14}$

\section{RESULTS}

Characteristics of the survey population. The mean age of individuals in this study was $49.8 \pm 7.3$ years (median age 50 years), of whom $69.3 \%$ were women (Table 1). Most of the participants possessed at least primary education $(2,882$ / $4,753 ; 60.6 \%)$ and were involved in agriculture-related occupations $(3,221 / 4,752 ; 67.8 \%)$.

Prevalence of HCV infection. The overall anti-HCV prevalence was $6.9 \%(327 / 4,769)$, which was higher among men $(71 \%, 233 / 327)$ than women $(29 \%, 94 / 327)$ (Table 1). Men were more likely to possess anti-HCV $(15.9 \%, 233 / 1,466)$ than women $(2.8 \%, 94 / 3,303)$ (Table $1, P<0.001)$. Seropositivity rates were significantly different among the 11 districts of Phetchabun and were generally higher in the northern districts than in the south $(P<0.001)$ (Table 2, Figure 1). The prevalence was highest in district $3(15.5 \%)$ and lowest in district 8 (0.9\%). Seropositivity also significantly differed among age-groups $(P=0.034)$ and was highest in adults aged $50-54$ years $(8.7 \%)$ (Table 1, Supplemental Table 2).

The majority of the $327 \mathrm{HCV}$-seropositive individuals had detectable HCV RNA (75.8\%, 248/327), and approximately three of four $(76.6 \%, 190 / 248)$ were men (Table 2$)$. In addition, $81.5 \%(190 / 233)$ of seropositive men had detectable HCV RNA compared with $61.7 \%$ (58/94) of women, which indicated active infection. Hepatitis B virus coinfection was found in $5.8 \%(19 / 327)$ and $4 \%(10 / 248)$ of individuals with HCV seropositive and active infection, respectively.

Genetic distribution of HCV genotypes. From $248 \mathrm{HCV}$ sequences identified from HCV RNA-positive samples, phylogenetic analysis with reference strains showed that $27.8 \%$ (69/248) were genotype 1 (1a: $22.4 \%$ or $56 / 248,1$ b: $5.2 \%$ or 13/248), $31.5 \%$ (78/248) were genotype 3 (3a: $30 \%$ or $75 / 248$, 3b: $1.2 \%$ or $3 / 248)$, and $40.7 \%$ (101/248) were genotype 6 (6f: $34.4 \%$ or $86 / 248,6 \mathrm{i}: 2.0 \%$ or $5 / 248,6$ n: $4.0 \%$ or $10 / 248$ ) (Figure 2).

Estimates of HCV seropositivity and viremia. Using the results from this study, we estimated the gender-adjusted 
TABLE 1

Demographic factors associated with anti-HCV seropositivity in this study

\begin{tabular}{|c|c|c|c|c|c|c|}
\hline \multirow[b]{2}{*}{ Variable } & \multirow[b]{2}{*}{ Numbers } & \multirow[b]{2}{*}{ Anti-HCV-positive (\%) } & \multicolumn{2}{|c|}{ Univariate analysis } & \multicolumn{2}{|c|}{ Multivariate analysis } \\
\hline & & & Odds ratio (95\% Cl) & $P$-value & Odds ratio $(95 \% \mathrm{Cl})$ & $P$-value \\
\hline $\mathrm{Age}^{\star}$ & $49.8(7.3)$ & & $1.2(1.0,1.4)$ & 0.010 & $1.1(0.9,1.3)$ & 0.517 \\
\hline Age-groups (years) $†$ & & & NA & NA & NA & NA \\
\hline $35-39$ & 446 & $23(5.2)$ & & & & \\
\hline $40-44$ & 757 & $42(5.5)$ & & & & \\
\hline $45-49$ & 1,087 & $64(5.9)$ & & & & \\
\hline $50-54$ & 1,108 & $96(8.7)$ & & & & \\
\hline $55-59$ & 868 & $64(7.4)$ & & & & \\
\hline $60-64$ & 503 & $38(7.6)$ & & & & \\
\hline Total & 4,769 & 327 (6.9) & & & & \\
\hline Gender & & & $6.5(5.0,8.3)$ & $<0.001$ & $6.3(4.9,8.1)$ & $<0.001$ \\
\hline Male & 1,466 & $233(15.9)$ & & & & \\
\hline Female & 3,303 & $94(2.8)$ & & & & \\
\hline Geographic residence & & & $4.1(3.1,5.4)$ & $<0.001$ & $4.0(3.0,5.3)$ & $<0.001$ \\
\hline North $\ddagger$ & 2,396 & $259(10.8)$ & & & & \\
\hline South§ & 2,373 & $68(2.9)$ & & & & \\
\hline \multicolumn{7}{|l|}{ Education attained } \\
\hline$\leq$ Grade 6 & 2,882 & $230(8.0)$ & $2.0(1.4,2.7)$ & $<0.001$ & $1.7(1.2,2.4)$ & 0.003 \\
\hline Grade 7-9 & 643 & $45(7.0)$ & $1.7(1.1,2.6)$ & 0.011 & $1.5(1.0,2.4)$ & 0.050 \\
\hline$\geq$ Grade 10 & 1,228 & $52(4.2)$ & $1(0)$ & & $1(0)$ & \\
\hline Total & 4,753 & 327 (6.9) & & & & \\
\hline \multicolumn{7}{|l|}{ Occupation } \\
\hline Agriculture & 3,221 & $241(7.5)$ & $2.5(1.6,4.0)$ & $<0.001$ & $1.9(1.2,3.0)$ & 0.009 \\
\hline Temporary employee & 816 & $64(7.8)$ & $2.7(1.6,4.4)$ & $<0.001$ & $2.1(1.2,3.5)$ & 0.006 \\
\hline \multirow[t]{2}{*}{ Non-agriculture } & 715 & $22(3.1)$ & $1(0)$ & & $1(0)$ & \\
\hline & 4,752 & 327 (6.9) & & & & \\
\hline
\end{tabular}

prevalence of anti-HCV and active HCV infection to capture the burden of HCV in Phetchabun (Table 3). Among the population ages 35-65 years in which there were 451,631 residents (according to the 2019 population census registry), we determined that approximately 38,502 (8.5\%) of Phetchabun residents in this age-group possess anti-HCV, of whom $82.8 \%(31,881 / 38,502)$ are men. In addition, we calculated that 30,239 or $6.7 \%$ of the residents are currently infected with $\mathrm{HCV}$, the overwhelming majority of whom are men $(86.5 \%$, $26,143 / 30,239)$. Thus, men are roughly five times $(31,881$ : $6,621)$ more likely to be HCV seropositive and 6.4 times
$(26,143: 4,096)$ more likely to have active HCV infection than women in this region.

Factors associated with HCV infection. We examined factors associated with HCV infection using logistic regression to elucidate the relationships between anti-HCVreactive samples with age, gender, geography, education attained, and occupation. By univariate analysis, the antiHCV-positive individuals were more likely to be older $(P=$ $0.010)$, men $(P<0.001)$, residing in northern Phetchabun $(P<$ $0.001)$, possessed less than grade-10 education $(P<0.012)$, and engaged in either agriculture $(P<0.001)$, or were temporarily

TABLE 2

HCV prevalence and HBV coinfection in Phetchabun Province

\begin{tabular}{|c|c|c|c|c|c|c|c|c|c|c|c|}
\hline \multirow[b]{2}{*}{ District } & \multicolumn{3}{|c|}{ Number of samples } & \multicolumn{3}{|c|}{ Anti-HCV-positive (\%) } & \multicolumn{3}{|c|}{ HCV RNA positive (\%) } & \multicolumn{2}{|c|}{ HBVsAg positive (\%) } \\
\hline & Men & Women & Total & Men & Women & Total $^{*}$ & Men & Women & Total & Anti-HCV† & HCV RNA \\
\hline 1 & 270 & 789 & 1,059 & $61(22.6)$ & $31(3.9)$ & $92(8.7 \%)$ & 53 (69.7) & $23(30.3)$ & $76(82.6)$ & $5(5.4)$ & $4(5.3)$ \\
\hline 2 & 136 & 220 & 356 & $10(7.4)$ & $6(2.7)$ & $16(4.5)$ & $8(72.7)$ & $3(27.3)$ & $11(68.8)$ & $2(12.5)$ & $1(9.1)$ \\
\hline 3 & 361 & 430 & 791 & $105(29.1)$ & $18(4.2)$ & $123(15.5)$ & 85 (87.6) & 12 (12.4) & 97 (77.2) & $8(6.5)$ & $4(4.1)$ \\
\hline 4 & 75 & 228 & 303 & 17 (22.7) & $10(4.4)$ & 27 (8.9) & 16 (69.6) & 7 (30.4) & 23 (85.2) & $1(3.7)$ & $0(0.0)$ \\
\hline 5 & 172 & 428 & 600 & $8(4.7)$ & $6(1.4)$ & $14(2.3)$ & $6(66.7)$ & 3 (33.3) & $9(64.3)$ & $2(14.2)$ & $1(11.1)$ \\
\hline 6 & 83 & 256 & 339 & 1 (1.2) & $4(1.6)$ & $5(1.5)$ & $0(0.0)$ & $2(100)$ & $2(40.0)$ & $0(0.0)$ & $0(0.0)$ \\
\hline 7 & 146 & 400 & 546 & 17 (11.6) & $8(2.0)$ & $25(4.6)$ & $12(75.0)$ & $4(25.0)$ & $16(64.0)$ & $0(0.0)$ & $0(0.0)$ \\
\hline 8 & 106 & 243 & 349 & $1(0.9)$ & $2(0.8)$ & $3(0.9)$ & $0(0.0)$ & $1(100.0)$ & 1 (33.3) & $0(0.0)$ & $0(0.0)$ \\
\hline 9 & 28 & 61 & 89 & $3(10.7)$ & $3(4.9)$ & $6(6.7)$ & $2(66.7)$ & $1(33.3)$ & $3(50.0)$ & $0(0.0)$ & $0(0.0)$ \\
\hline 10 & 36 & 147 & 183 & $1(2.8)$ & $4(2.7)$ & $5(2.7)$ & $1(100.0)$ & $0(0.0)$ & $1(20.0)$ & $0(0.0)$ & $0(0.0)$ \\
\hline 11 & 53 & 101 & 154 & $9(17.0)$ & $2(2.0)$ & $11(7.1)$ & 7 (77.8) & 2 (22.2) & 9 (81.8) & $1(9.1)$ & $0(0.0)$ \\
\hline Total & 1,466 & 3,303 & 4,769 & 233 (15.9) & $94(2.8)$ & 327 (6.9) & 190 (76.6) & $58(23.4)$ & $248(75.8)$ & 19 (5.8)‡ & $10(4.0) \S$ \\
\hline
\end{tabular}

$P$-value $<0.001$ (categorical analysis).

I Percentage calculated based on anti-HCV-positive sample $(N=327)$.

$\S$ Percentage calculated based on HCV RNA-positive sample $(N=248)$. 


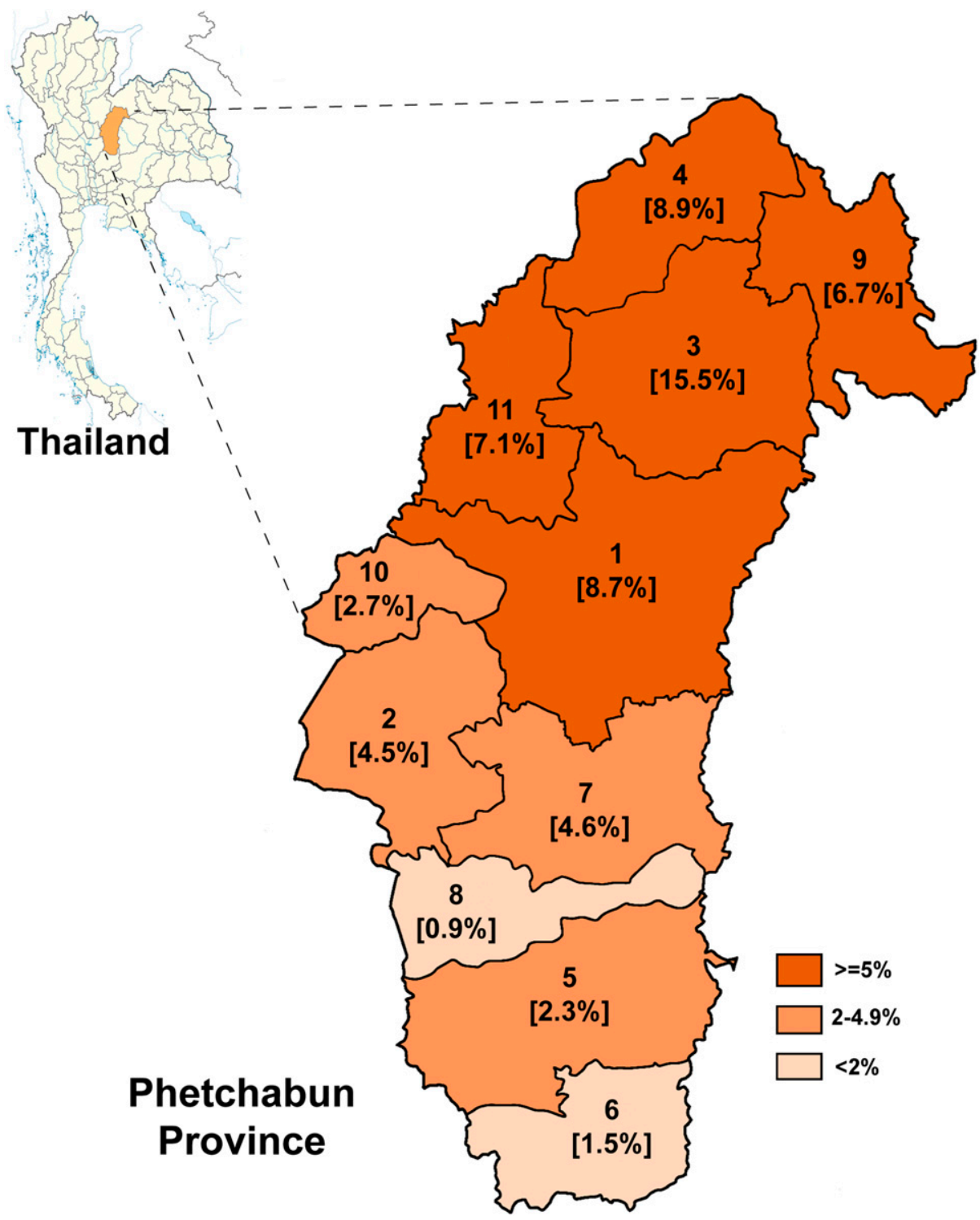

FIGURE 1. Hepatitis C virus (HCV) seroprevalence in 11 districts of Phetchabun Province. Approximately $1 \%$ of randomly represented adults aged 35-64 years were tested for anti-HCV. The district numbers and their corresponding seroprevalence rates are shown. This figure appears in color at www.ajtmh.org.

employed $(P<0.001)$ (Table 1). After adjusting for age, the multivariate analysis confirmed that being men $(P<0.001$, adjusted OR $6.3,95 \% \mathrm{Cl}$ : 4.9-8.1), residents of northern Phetchabun $(P<0.001$, adjusted OR $4.0,95 \% \mathrm{Cl}: 3.0-5.3)$, primary education $(P=0.003$, adjusted OR $1.7,95 \% \mathrm{Cl}$ : 1.2-2.4) and occupation in agriculture $(P=0.009$, adjusted $O R$ $1.9,95 \% \mathrm{Cl}: 1.2-3.0)$ or temporary worker $(P=0.006$, adjusted OR 2.1, 95\% Cl: 1.2-3.5) were independently associated with HCV seropositivity.

\section{DISCUSSION}

In this study, we conducted a comprehensive assessment of the HCV burden in an endemic region of Thailand. Although the overall HCV seroprevalence in Phetchabun was 6.9\%, which was already considerably higher than the national average of $0.9 \%$, evidence of past HCV infection was as high as $15.5 \%$ in some places. ${ }^{5,8,15}$ We estimated that as many as $6.7 \%$ of residents in this region may be actively infected with $\mathrm{HCV}$, far higher than the $0.4 \%$ nationally..$^{5}$ Most are men engaged in agricultural work who may not be aware of their hepatitis $\mathrm{C}$ status because of limited educational background.

Hepatitis $C$ virus infection is not vaccine preventable and remains substantial in many developing countries with emerging economies and in the population of workingage. ${ }^{16,17}$ In Southeast Asia alone, it is estimated that at least 4.7 million people may harbor $\mathrm{HCV} .{ }^{1}$ Almost half a million Thais may have chronic $\mathrm{HCV}$ infection and will require treatment in the near future to prevent the potential development of liver cirrhosis and hepatocellular carcinoma. ${ }^{1,5}$ This endeavor poses logistical, budgetary, and healthcare constraints because latent HCV infection is asymptomatic and patients are unlikely to be aware of their infection status. Focusing on the population-wide screening of individuals most likely to be at risk of infection by geographical region or demographic 


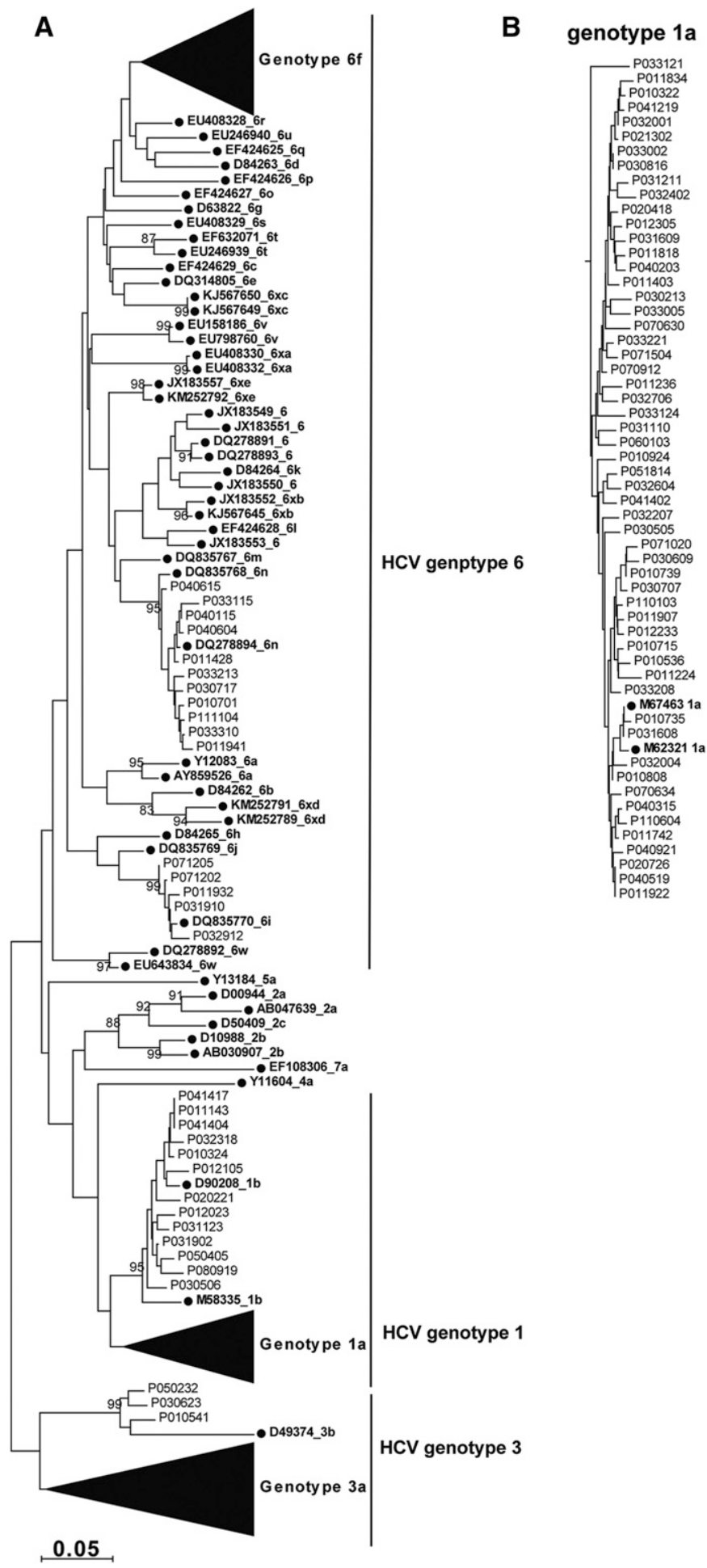

C

genotype 3a

D genotype 6f

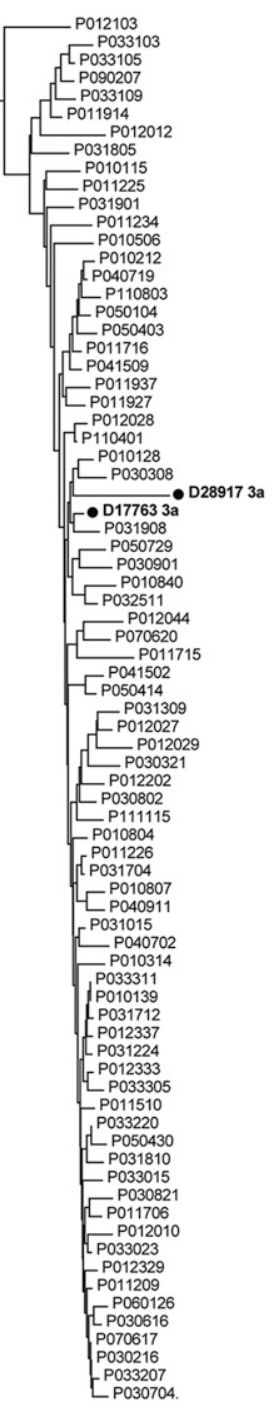

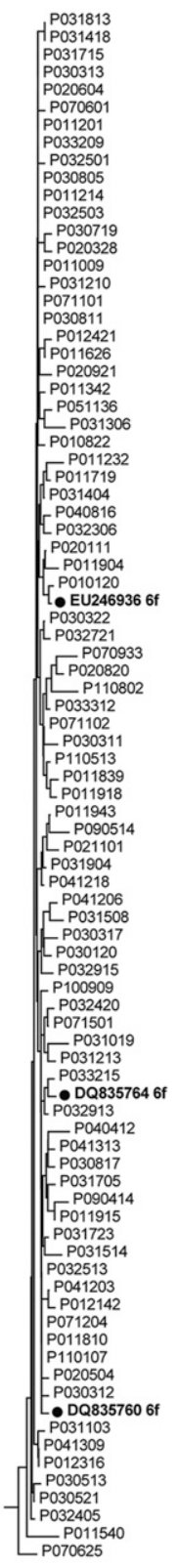

FIGURE 2. Phylogenetic analysis of the partial core gene sequences identified from hepatitis $C$ virus-positive individuals. (A) The neighbor-joining tree was constructed using Kimura's two-parameter nucleotide substitution model with 1,000 bootstraps. References sequences are dotted $(\bullet)$. Condensed clusters of genotype 1a (B), genotype 3a (C), and genotype $6 f(\mathbf{D})$ are shown.

group, termed micro-elimination, may be more feasible before nationwide implementation. ${ }^{18-20}$ Targeted diagnosis and delivery of HCV treatment regionally, as opposed to referrals to distantly located physician specialists, are anticipated to be more expeditious and cost-effective to initiate. As such, establishing the epidemiological baseline is crucial to assess the regional burden of $\mathrm{HCV}$ infection, especially in some communities with limited healthcare infrastructure.

Targeting a subpopulation with the highest HCV prevalence for treatment with DAAs has been proposed to be a feasible strategy toward an overall elimination of viral hepatitis, especially in countries with emerging economies. ${ }^{18,21,22}$ As such, 
TABLE 3

Estimated HCV antibody and RNA positivity among Phetchabun residents

\begin{tabular}{|c|c|c|c|c|c|c|c|c|c|c|c|}
\hline \multirow[b]{2}{*}{ District } & \multirow{2}{*}{$\frac{\% \text { Anti-HCV }}{\text { Male/female }}$} & \multirow{2}{*}{$\frac{\% \text { HCV RNA }}{\text { Male/female }}$} & \multicolumn{3}{|c|}{ Target population* } & \multicolumn{3}{|c|}{$\begin{array}{l}\text { Estimated HCV-seropositive } \\
\text { population } \\
\end{array}$} & \multicolumn{3}{|c|}{$\begin{array}{l}\text { Estimated HCV viremic } \\
\text { population }\end{array}$} \\
\hline & & & Male & Female & Total & Male & Female & Total & Male & Female & Total \\
\hline 1 & $22.6 / 3.9$ & $86.9 / 74.2$ & 46,105 & 49,999 & 96,104 & 10,420 & 1,950 & 12,370 & 9,053 & 1,447 & 10,500 \\
\hline 2 & $7.4 / 2.7$ & $80.0 / 50.0$ & 17,796 & 18,241 & 36,037 & 1,317 & 493 & 1,809 & 1,054 & 246 & 1,300 \\
\hline 3 & $29.1 / 4.2$ & $81.0 / 66.7$ & 35,014 & 37,266 & 72,280 & 10,189 & 1,565 & 11,754 & 8,248 & 1,043 & 9,291 \\
\hline 4 & $22.7 / 4.4$ & $94.1 / 70.0$ & 14,887 & 15,307 & 30,194 & 3,379 & 674 & 4,053 & 3,181 & 471 & 3,652 \\
\hline 5 & $4.7 / 1.4$ & $75.0 / 50.0$ & 29,389 & 30,967 & 60,356 & 1,381 & 434 & 1,815 & 1,036 & 217 & 1,253 \\
\hline 6 & $1.2 / 1.6$ & $0.0 / 50.0$ & 15,658 & 16,373 & 32,031 & 188 & 262 & 450 & 0 & 131 & 131 \\
\hline 7 & $11.6 / 2$ & $70.6 / 50.0$ & 25,372 & 26,175 & 51,547 & 2,943 & 524 & 3,467 & 2,078 & 262 & 2,340 \\
\hline 8 & $0.9 / 0.8$ & $0.0 / 50.0$ & 15,977 & 16,736 & 32,713 & 144 & 134 & 278 & 0 & 67 & 67 \\
\hline 9 & $10.7 / 4.9$ & 66.7/33.3 & 4,449 & 4,130 & 8,579 & 476 & 202 & 678 & 317 & 67 & 384 \\
\hline 10 & $2.8 / 2.7$ & $100.0 / 0.0$ & 8,580 & 8,876 & 17,456 & 240 & 240 & 480 & 240 & 0 & 240 \\
\hline 11 & $17.0 / 2.0$ & $77.8 / 100.0$ & 7,080 & 7,254 & 14,334 & 1,204 & 145 & 1,349 & 936 & 145 & 1,081 \\
\hline Total & $15.9 / 2.8$ & $81.5 / 61.7$ & 220,307 & 231,324 & 451,631 & 31,881 & 6,621 & 38,502 & 26,143 & 4,096 & 30,239 \\
\hline
\end{tabular}

${ }^{\star}$ Target population data were extrapolated from the 2019 population census registry (for age 35-65 years) (cited: September 20, 2019). ${ }^{11}$

Phetchabun is an ideal candidate for HCV micro-elimination owing to the availability of essential epidemiological baseline, defined demographics and healthcare infrastructure, and ongoing disease surveillance. ${ }^{9,10}$ From this study, most active $\mathrm{HCV}$ infection occurs in men of working age who are the primary wage earners and identify residences in northern domicile, agriculture or temporary work, and primary education as risk factors. Micro-elimination programs have successfully been implemented and have led to significant reductions in chronic hepatitis $\mathrm{C}$ infection in several countries, including Australia, Spain, Portugal, Iceland, Georgia, and Egypt. ${ }^{18,22}$ The inclusion of targeted populations at high risk of HCV has involved region-based or demographic vulnerability. This strategy has been helped by several factors. First, there is a tendency for a natural decline in new HCV infection as a result of mortality associated with hepatitis due to an aging population. Second, some illicit drugs have supplanted intravenous drug use, which is a major risk factor for this blood-borne pathogen. Third, better and improved screening of blood donations have now become the standard even for many resource-limited settings. These factors have been seen in Thailand where surveillance among Thai blood donors and the general population showed a decrease in HCV carrier rate from $\sim 2 \%$ to $<1 \%$ in recent years. ${ }^{5}$ Furthermore, it is projected that a further reduction is expected in the next 20 years in which untreated HCV infection could account for less than $0.2 \%$. Finally, major advances in the development and extensive use of DAAs would be expected to further reduce the prevalence of infection to $<0.001 \%$ if prices become affordable. ${ }^{23}$

The current rate of high HCV infection in Phetchabun emphasizes the urgent need to initiate a test-to-treat program with pan-genotypic DAAs in Thailand. Unlike the rest of Thailand in which genotype $3 a$ is predominant, the high prevalence of genotype $6(41 \%)$ in Phetchabun affords an opportunity to further evaluate the efficacy of DAAs on this genotype because data from previous efficacy studies have been limited. Interestingly, genotype $6 a$ is common in southern China and Vietnam, whereas genotypes $6 p$ and $6 r$ are common in Cambodia. ${ }^{24}$ Elsewhere in the region, Laos also reported diverse subtypes for genotype 6, which includes genotype $6 \mathrm{~m}$ and $6 \mathrm{n}$ reported in Myanmar. ${ }^{25,26}$ By contrast, Malaysia reported few genotype 6 infection. ${ }^{27}$ Although the $\mathrm{HCV}$ treatment response rate has historically depended on the viral genotype and the types of the antiviral regimen, DAAs have now made that prerequisite increasingly obsolete. DAAs such as sofosbuvir-ledipasvir (SOF-LDV) yielded a $64 \%$ sustained virological response (SVR) at week 12 after treatment (SVR12) for genotype 3 and $100 \%$ if combined with ribavirin (RBV). ${ }^{28}$ Another study found that the SVR for SOFLDV was $100 \%$ with and $92 \%$ without RBV. ${ }^{29}$ The virological response rate of SOF-LDV treatment can sometimes be lower in patients with HCV genotypes $3(75.0 \%$ SVR12) and 6 (64.1\% SVR12) than genotype 1 (96.8\% SVR), whereas pan-genotypic treatment such as sofosbuvir-velpatasvir (SOF-VEL) yielded a high cure rate in patients infected with genotypes $3(97.4 \%)$ and $6(90.4 \%) .{ }^{30}$ In some studies, the SVR12 rate was up to $99 \%$ in patients treated with SOF-VEL (with or without RBV), regardless of HCV genotype. ${ }^{31}$ Similarly, sofosbuvir-daclatasvir pan-genotypic regimen resulted in $96.9 \%$ and $93.3 \%$ SVR for genotypes 3 and 6 , respectively. ${ }^{32}$ Thus, the high prevalence of HCV genotype 6 and to a lesser extent genotypes 3 and 1 from this study suggest that treatment with a pan-genotypic DAAs regimen is most appropriate and is expected to be highly safe and effective.

Increasing HCV diagnostic rates and treatment coverage in Thailand will be challenging for several reasons. Insufficient healthcare infrastructure where trained healthcare workers can accurately and quickly diagnose HCV infection especially in remote communities remains a major obstacle. Patients are sometimes expected to bear the cost of disease screening as the government neither provides reimbursements nor incentives toward medical fees associated with hepatitis treatment. The recent availability of generic DAAs in Thailand beginning in 2018 has enabled its inclusion into the national treatment guideline and its accessibility to a greater number of HCVinfected patients. Thus far, the fact that pan-genotypic DAAs are not currently the standard therapy in Thailand has been hindered by the lack of defined HCV epidemiological baseline, cost, and complexity in program implementation. To partially overcome these obstacles, HCV treatment at the local primary healthcare centers rather than referrals to specialists at larger hospitals should reduce the test-to-treat time and provide greater treatment access for HCV-infected Thai patients.

Decentralized HCV treatment and care have proven effective in several resource-limited countries. This approach, which allows primary care providers such as trained nurses, primary care physicians, and even pharmacists the ability to 
administer DAAs to patients, would greatly increase treatment access and unburden both the physician specialists and patients from treatment restrictions. Without a simplified diagnostic strategy, there is also a risk of reduced medication compliance and dose continuity leading to failed treatment plans. Any antiviral resistance would necessitate newer and possibly more expensive antivirals. The effectiveness of decentralized care was previously demonstrated by a study in Columbia, where they found no significant differences in the treatment outcome using SOF-LDV whether administered by nurse practitioners, point-of-care physicians, or specialists (84-89\%). ${ }^{33}$ Similarly, a study from Pakistan showed that with a simplified HCV testing, SOF-RBV therapy, and monitoring by local healthcare workers resulted in an SVR of $83 \%$ with a relatively low $10 \%$ loss to follow-up. ${ }^{34}$

Admittedly, this study has several limitations. Although attempts were made in choosing participants at random, selection bias cannot be ruled out because local healthcare workers were given the discretion in randomizing participant selection. There were more women who visited healthcare centers than men during the enrollment period. Our estimates of HCV focused on working-age adults and did not include those older than 64 years. Such inclusion might increase the overall prevalence and increase the number of adults requiring treatment. For these reasons, findings in Phetchabun may not be generalizable for the entire country. We used an automated HCV assay, which is not available or financially feasible for screening the population at large. It is known that some chronic HBV infections are coinfected with hepatitis $D$ virus. Epidemiological screening for the latter was not performed in this study, but continued decline in HBV infection in Thailand may obviate such need. Despite these limitations, this epidemiological study in Phetchabun can help guide policies toward managing $\mathrm{HCV}$ elimination and developing a costeffective population-based screening strategy. In the near future, the combined implementation of HCV screening using rapid diagnostic tests and initiating treatment at the point of care by local healthcare providers is foreseeable to achieve $\mathrm{HCV}$ elimination in many emerging economies.

Received November 1, 2019. Accepted for publication April 9, 2020.

Published online May 11, 2020.

Note: Supplemental tables appear at www.ajtmh.org.

Acknowledgments: We thank the staff at the health centers and hospitals, and residents of Phetchabun who made this study possible.

Financial support: This study was supported by the Chulalongkorn University Fund (CU GJF 610130 01), Research Chair Grant from the National Science and Technology Development Agency (P-15-50004), the Regional Health 2 Office Fund, the Center of Excellence in Clinical Virology of Chulalongkorn University and Hospital (GCE 59-00930005), and the Rachadapisek Sompote Fund of Chulalongkorn University for postdoctoral fellowship to Rujipat Wasitthankasem.

Authors' addresses: Rujipat Wasitthankasem, Center of Excellence in Clinical Virology, Faculty of Medicine, Chulalongkorn University, Bangkok, Thailand, National Biobank of Thailand, National Science and Technology Development Agency, Pathum Thani, Thailand, and National Center for Genetic Engineering and Biotechnology, National Science and Technology Development Agency, Pathum Thani, Thailand, E-mail: rujipat.was@biotec.co.th. Napaporn Pimsingh and Khuandao Treesun, Non-Communicable Disease Control Group, Phetchabun Provincial Health Office, Phetchabun, Thailand, E-mails: napaporn.tu16@gmail.com and khuandaonurse@gmail.com. Nawarat Posuwan, Preeyaporn Vichaiwattana, Chompoonut Auphimai, Ilada Thongpan, Sompong Vongpunsawad, and Yong Poovorawan, Center of Excellence in Clinical Virology, Faculty of Medicine, Chulalongkorn University, Bangkok, Thailand, E-mails: nawarat.po@gmail.com, preeya teiy@hotmail.com, chompoonut.bit@hotmail.com, ilada.cu09@gmail. com, sompong.vo@chula.ac.th, and yong.p@chula.ac.th. Sissades Tongsima, National Biobank of Thailand, National Science and Technology Development Agency, Pathum Thani, Thailand, and National Center for Genetic Engineering and Biotechnology, National Science and Technology Development Agency, Pathum Thani, Thailand, E-mail: sissades@biotec.or.th.

\section{REFERENCES}

1. Polaris Observatory HCV Collaborators, 2017. Global prevalence and genotype distribution of hepatitis $C$ virus infection in 2015: a modelling study. Lancet Gastroenterol Hepatol 2: 161-176.

2. Thomas DL, 2019. Global elimination of chronic hepatitis. N Engl J Med 380: 2041-2050.

3. World Health Organization, 2016. Combating Hepatitis $B$ and $C$ to Reach Elimination by 2030. Available at: https://www.who.int/ hepatitis/publications/hep-elimination-by-2030-brief/en/. Accessed October 30, 2019.

4. Gower E, Estes C, Blach S, Razavi-Shearer K, Razavi H, 2014. Global epidemiology and genotype distribution of the hepatitis C virus infection. J Hepatol 61 (Suppl 1): S45-S57.

5. Wasitthankasem R et al., 2016. Decreasing hepatitis $C$ virus infection in Thailand in the past decade: evidence from the 2014 national survey. PLoS One 11: e0149362.

6. Posuwan N, Vuthitanachot V, Chinchai T, Wasitthankasem R, Wanlapakorn N, Poovorawan Y, 2019. Serological evidence of hepatitis $A, B$, and $C$ virus infection in older adults in Khon Kaen, Thailand and the estimated rates of chronic hepatitis $B$ and $C$ virus infection in Thais, 2017. PeerJ 7: e7492.

7. Chimparlee N, Oota S, Phikulsod S, Tangkijvanich P, Poovorawan $Y, 2011$. Hepatitis $B$ and hepatitis $C$ virus in Thai blood donors. Southeast Asian J Trop Med Public Health 42: 609-615.

8. Wasitthankasem R et al., 2017. Assessment of hepatitis $C$ virus infection in two adjacent Thai provinces with drastically different seroprevalence. PLoS One 12: e0177022.

9. Wasitthankasem $\mathrm{R}$ et al., 2018. Liver disease burden and required treatment expenditures for hepatitis $\mathrm{C}$ virus $(\mathrm{HCV})$ infection in Thailand: implications for HCV elimination in the new therapeutic era, a population-based study. PLoS One 13: e0196301.

10. Wasitthankasem Ret al., 2018. Birth-cohort HCV screening target in Thailand to expand and optimize the national HCV screening for public health policy. PLoS One 13: e0202991.

11. Official Statistics Registration Systems, 2019. Population Registry, Phetchabun Province. Available at: http://stat.dopa.go.th/ stat/statnew/upstat_age.php. Accessed October 30, 2019.

12. Phetchabun Provincial Office, 2020. Province Information. Available at: http://www.phetchabun.go.th/data_detail.php?content_ id=3. Accessed February 3, 2020.

13. Smith DB, Bukh J, Kuiken C, Muerhoff AS, Rice CM, Stapleton JT, Simmonds $P, 2014$. Expanded classification of hepatitis $C$ virus into 7 genotypes and 67 subtypes: updated criteria and genotype assignment web resource. Hepatology 59: 318-327.

14. Data Health Center, 2019. Population Registration of Phetchabun Province 2019. Thailand: Phetchabun Public Health Office, Ministry of Public Health. Available at: https://pnb.hdc.moph.go.th/ hdc/main/index_pk.php. Accessed September 20, 2019.

15. Buathong $R, 2013$. Risk factors of viral hepatitis $C$ infection among adults in a northern province, Thailand, 2006. Wkly Epidemiol Surveill Rep 44: S47-S56.

16. Geboy AG, Mahajan S, Daly AP, Sewell CF, Fleming IC, Cha HA, Perez IE, Cole CA, Ayodele AA, Fishbein DA, 2016. High hepatitis $C$ infection rate among baby boomers in an urban primary care clinic: results from the HepTLC initiative. Public Health Rep 131: 49-56.

17. Smith BD et al.; Centers for Disease Control Prevention, 2012. Recommendations for the identification of chronic hepatitis $C$ virus infection among persons born during 1945-1965. MMWR Recomm Rep 61: 1-32.

18. Lazarus JV et al., 2018. The Micro-elimination approach to eliminating hepatitis C: strategic and operational considerations. Semin Liver Dis 38: 181-192. 
19. Morgan JR, Servidone M, Easterbrook P, Linas BP, 2017. Economic evaluation of HCV testing approaches in low- and middle-income countries. BMC Infect Dis 17: 697.

20. World Health Organization, 2017. Guidelines on Hepatitis $B$ and $C$ Testing. Available at: https://www.who.int/hepatitis/publications/ guidelines-hepatitis-c-b-testing/en/. Accessed March 10, 2020.

21. Taye BW, 2019. A path to ending hepatitis $C$ in Ethiopia: a phased public health approach to achieve micro-elimination. Am J Trop Med Hyg 101: 963-972.

22. Shiha G, Metwally AM, Soliman R, Elbasiony M, Mikhail NNH, Easterbrook P, 2018. An educate, test, and treat programme towards elimination of hepatitis C infection in Egypt: a community-based demonstration project. Lancet Gastroenterol Hepatol 3: 778-789.

23. Stvilia $\mathrm{K}$ et al., 2019. Progress in testing for and treatment of hepatitis $C$ virus infection among persons who inject drugs Georgia, 2018. Morb Mortal Wkly Rep 68: 637-641.

24. Poovorawan K, Pan-Ngum W, White LJ, Soonthornworasiri N, Wilairatana P, Wasitthankasem $\mathrm{R}$, Tangkijvanich $\mathrm{P}$, Poovorawan $Y, 2016$. Estimating the impact of expanding treatment coverage and allocation strategies for chronic hepatitis $C$ in a direct antiviral agent era. PLoS One 11: e0163095.

25. Nouhin $\mathrm{J}$ et al., 2019. Molecular epidemiology of hepatitis $C$ virus in Cambodia during 2016-2017. Sci Rep 9: 7314.

26. Hubschen JM, Jutavijittum $P$, Thammavong $T$, Samountry $B$, Yousukh A, Toriyama K, Sausy A, Muller CP, 2011. High genetic diversity including potential new subtypes of hepatitis $C$ virus genotype 6 in Lao People's Democratic Republic. Clin Microbiol Infect 17: E30-E34.

27. Akkarathamrongsin S, Praianantathavorn $\mathrm{K}$, Hacharoen $\mathrm{N}$, Theamboonlers A, Tangkijvanich P, Poovorawan Y, 2011. Seroprevalence and genotype of hepatitis $C$ virus among immigrant workers from Cambodia and Myanmar in Thailand. Intervirology 54: 10-16.
28. Ho SH, Ng KP, Kaur H, Goh KL, 2015. Genotype 3 is the predominant hepatitis $\mathrm{C}$ genotype in a multi-ethnic Asian population in Malaysia. Hepatobiliary Pancreat Dis Int 14: 281-286.

29. Gane EJ, Hyland RH, An D, Svarovskaia E, Pang PS, Brainard D, Stedman CA, 2015. Efficacy of ledipasvir and sofosbuvir, with or without ribavirin, for 12 weeks in patients with HCV genotype 3 or 6 infection. Gastroenterology 149: 1454-1461.

30. Dhiman RK, Grover GS, Premkumar M, Taneja S, Duseja A, Arora S, Rathi S, Satsangi S, Roy A, Investigators M, 2019. Decentralized care with generic direct-acting antivirals in the management of chronic hepatitis $\mathrm{C}$ in a public health care setting. J Hepatol 71: 1076-1085.

31. Hlaing NKT, Mitrani RA, Aung ST, Phyo WW, Serper M, Kyaw AMM, Bwa AH, Win KM, Reddy KR, 2017. Safety and efficacy of sofosbuvir-based direct-acting antiviral regimens for hepatitis $C$ virus genotypes 1-4 and 6 in Myanmar: real-world experience. J Viral Hepat 24: 927-935.

32. Bwa AH et al., 2019. Strategy and efficacy of generic and pangenotypic sofosbuvir/velpatasvir in chronic hepatitis $\mathrm{C}$ virus: a Myanmar experience. J Clin Exp Hepatol 9: 283-293.

33. Hlaing NKT et al., 2019. High sustained virologic response in genotypes 3 and 6 with generic NS5A inhibitor and sofosbuvir regimens in chronic HCV in Myanmar. $J$ Viral Hepat 26: 1186-1199.

34. Kattakuzhy $S$ et al., 2017. Expansion of treatment for hepatitis $C$ virus infection by task shifting to community-based nonspecialist providers: a nonrandomized clinical trial. Ann Intern Med 167: 311-318.

35. Capileno YA, Van den Bergh R, Donchunk D, Hinderaker SG, Hamid S, Auat R, Khalid GG, Fatima R, Yaqoob A, Van Overloop $C, 2017$. Management of chronic hepatitis $C$ at a primary health clinic in the high-burden context of Karachi, Pakistan. PLOS One 12: e0175562. 